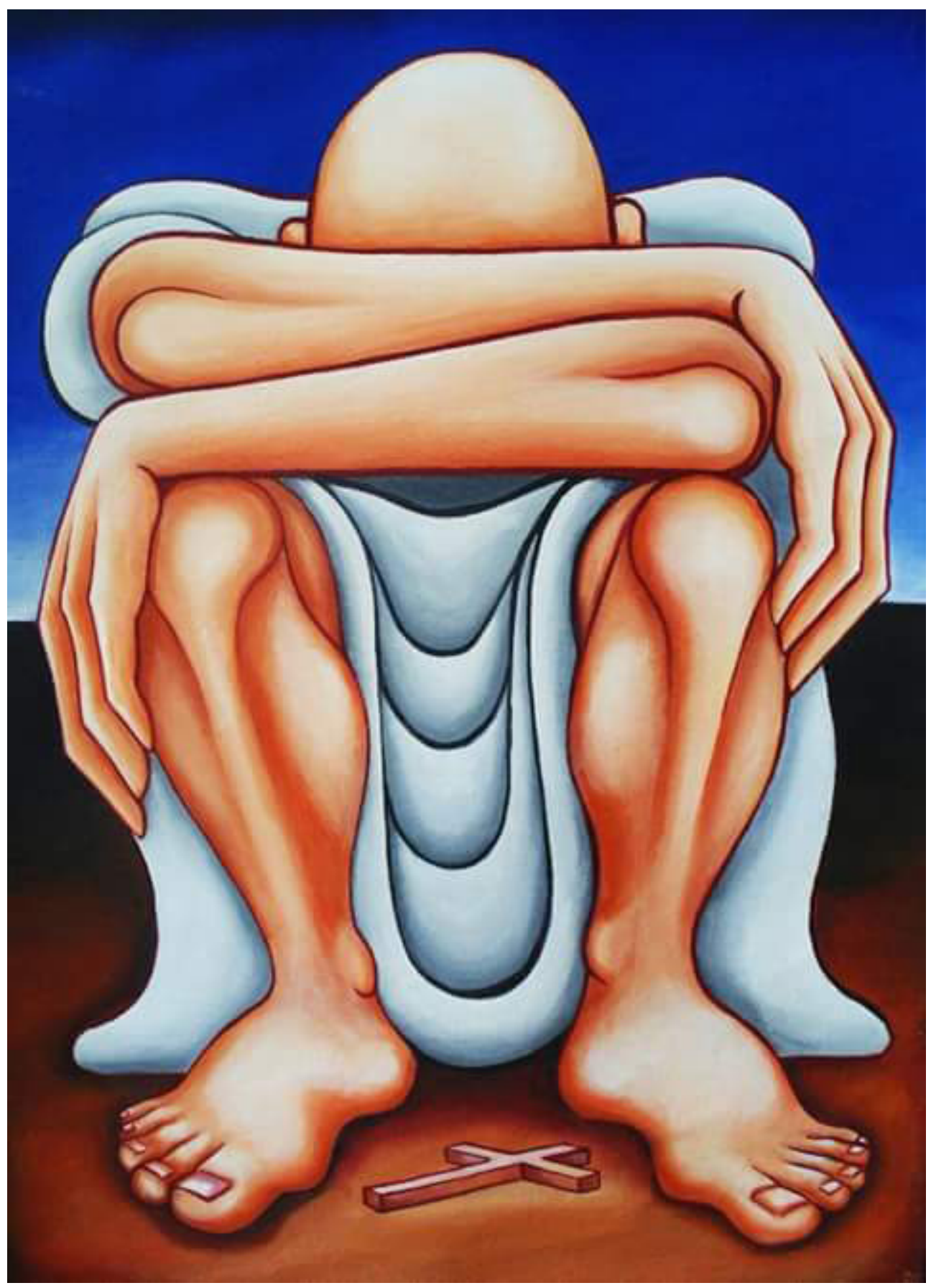

Artista: Heri Tapia 


\title{
Experiencia de innovación: “Club de astronomía Orión: el conocimiento del universo al alcance de tus manos"
}

\author{
EXPERIENCING INNOVATION: "THE ORION ASTRONOMY CLUB: KNOWLEDGE \\ OF THE UNIVERSE WITHIN YOUR REACH" \\ EXPERIÊNCIA DE INOVAÇÃO: "CLUBE DE ASTRONOMIA ORION: O \\ CONHECIMENTO DO UNIVERSO AO ALCANCE DAS SUAS MÃOS"
}

\author{
Liliana Gavilán Infante1/ lili_gavi@yahoo.es \\ Elizabeth Hernández Pérez²/ eliperh@yahoo.es \\ Luz Elena López Salas3 / neguslopezsalas@hotmail.com \\ Pedro Rivera Prieto4 / dertodzod@gmail.com
}

\begin{abstract}
Resumen
El incluir en la escuela el estudio de la astronomía, mostrándoles a los estudiantes cómo en esta disciplina se vincula diferentes áreas de conocimiento, es la idea inicial con la que en el año 2003, en el colegio El Rodeo, nace un club que vincula a un grupo de maestros, niños y jóvenes con un interés común, conocer sobre el universo. Se establece este tipo de asociación porque permite el desarrollo de un ambiente de aprendizaje colaborativo entre sus integrantes sin desligarla del contexto académico, haciéndola atractiva a los estudiantes.
\end{abstract}

Es una experiencia orientada, desde la astronomía, a contribuir en el mejoramiento del aprendizaje al desarrollar habilidades de pensamiento como observación, experimentación, análisis y argumentación en los estudiantes, sin distinguir niveles académicos ni áreas del conocimiento, por estar diseñada para trascender el aula, el currículo, los grados escolares y la jornada académica. Esto se logra, metodológicamente, al tomarse como referente la enseñanza basada en la indagación y al orientarse al desarrollo de proyectos por parte de los estudiantes con la asesoría de los maestros; didácticamente, se materializa, al utilizar múltiples estrategias tal como talleres, conferencias, video - foros, vinculación a proyectos institucionales e internacionales, ferias, actividades lúdicas, observaciones diurnas y nocturnas, uso de software astronómico, entre otras.
Palabras clave

Astronomía, aprendizaje autónomo, trabajo por proyectos, trabajo colaborativo, liderazgo, metodología basada en la indagación.

1 Licenciada en Biología de la Universidad Distrital Francisco José de Caldas. Especialista en Gerencia de Proyectos Educativos de la misma universidad. Magíster en Psicopedagogía de la Universidad de Barcelona. Coordinadora del Colegio El Rodeo. Gestora de la presente propuesta de innovación.

2 Licenciada en Matemáticas de la Universidad Antonio Nariño. Especialista en Aprendizaje escolar y sus dificultades de la Universidad Cooperativa de Colombia. Maestra de matemáticas del Colegio El Rodeo, se vincula al proyecto en el año 2004.

3 Licenciada en Preescolar de la Universidad Inpahu. Maestra de primaria del Colegio El Rodeo, se vincula al proyecto en el año 2010.

4 Licenciado en Diseño Tecnológico de la Universidad Pedagógica Nacional. Magíster en Educación de la Universidad Distrital Francisco José de Caldas. Maestro de Tecnología del Colegio El Rodeo, se vincula al proyecto en el año 2008. 


\section{Abstract}

The inclusion of the study of astronomy in school activities, showing students how this discipline links together different areas of knowledge, was the initial idea behind the creation of a club in 2003, in the El Rodeo School, which would gather a group of teachers, children, and young students with a common interest: to learn about the universe. This type of association was established because it allowed for the development of a collaborative learning environment among its members without separating it from the academic context, which made it attractive to students.

It is an experience, which is oriented, by focusing on astronomy, to contribute to the improvement of learning and the development of thinking skills in students, such as observation, experimentation, analysis, and argumentation, without distinguishing between academic levels and areas of knowledge, thanks to its specific design that transcends classroom, curriculum, school grades, and school hours. This is accomplished, in terms of methodology, by taking inquiry-based teaching as a reference point and by developing projects by students with advice from teachers. Didactically, it materializes through multiple strategies such as workshops, conferences, video-forums, participation in institutional and international projects, science fairs, recreational activities, day and night observations, and the use of astronomical softwares, among others.

\section{Resumo}

Incluir na escola o estudo da astronomia, mostrando para os estudantes como essa disciplina está vinculada a várias áreas do conhecimento, foi o principal objetivo com o qual, no ano 2003, no colégio El Rodeo, foi criado um clube que une um grupo de professores, crianças e jovens com um interesse comum: conhecer sobre o universo. Estabeleceu-se esse tipo de associação porque permite o desenvolvimento de um ambiente de aprendizagem colaborativa para seus membros sem desvinculá-la do contexto acadêmico, fazendo-a, assim, atrativa para os estudantes. É uma experiência orientada, desde a astronomia, para contribuir para o melhoramento da aprendizagem ao desenvolver habilidades de pensamento como observação, experimentação, análise e argumentação nos estudantes, sem diferenciar níveis acadêmicos nem áreas do conhecimento por estar concebida para transcender a aula, o currículo, os níveis escolares e o horário de estudos. Isso é possível, metodologicamente, por tomar como referência o ensino baseado na indagação e por estar orientada para o desenvolvimento de projetos por parte dos estudantes com a assessoria dos professores; didaticamente, é possível graças a múltiplas estratégias como oficinas, conferências, vídeofóruns, vinculação a projetos institucionais e internacionais, feiras, atividades lúdicas, observações diurnas e noturnas, uso de software astronômico, entre outras.

\section{Keywords}

Astronomy, autonomous learning, project-based work, collaborative work, leadership, inquiry-based methodology.

\section{Palavras chave}

Astronomia, aprendizagem autónoma, trabalho por projetos, trabalho colaborativo, liderança, metodologia baseada na indagação. 


\section{El nacimiento de una experiencia astronómica}

En el año 2003, Maloka y la Universidad de Valencia desarrollaron un seminario de Astrobiología en el cual participó la única maestra de biología del colegio el Rodeo, de esa época. Como resultado de este proceso, y al evidenciar que el estudio de la astronomía en la escuela estaba relegado a una unidad temática de las ciencias sociales, le surgió el interés de incluir en sus clases temas relacionados con el universo y la vida. Así, a partir de las inquietudes que tenían algunos estudiantes del grado séptimo de ese año sobre diferentes fenómenos astronómicos, inició un trabajo de aula con actividades específicas, lo cual culminó con una feria que dio cuenta de las indagaciones realizadas por ellos.

En 2004, con el creciente compromiso de varios estudiantes de octavo y noveno grado, decidió conformar un club que reuniera a quienes estuvieran interesados en conocer sobre el universo y demostrarles que la astronomía permite integrar diferentes áreas del conocimiento que podrían contribuir con su formación académica. A mediados de este año se unió la maestra de Matemáticas y, tres años después, se integraron al equipo de maestros coordinadores del club, el profesor de tecnología e informática y la profesora de primaria.

Hoy por hoy, el club se ha transformado y una de las preocupaciones actuales es que, debido a la parcialización de la enseñanza por especialidades, se genera conflicto en los estudiantes a la hora de relacionar los saberes que cada área del conocimiento les brinda; por eso, y considerando que la astronomía tiene vínculo con tantas disciplinas, surge el interrogante de cómo lograr que los integrantes del club utilicen esta interdisciplinariedad para la articulación de los aprendizajes obtenidos durante su proceso de formación y la potenciación de sus habilidades de pensamiento.

\section{Características de los participantes (estudiantes)}

La experiencia involucraba a estudiantes del ciclo II al $\checkmark$ del Colegio El Rodeo ubicado en la parte media de la localidad cuarta-San Cristóbal. A partir del segundo semestre del año 2013, se decidió vincular a los niños de primer ciclo.
A pesar de las diferentes transformaciones suscitadas en la estructura de las familias, los estudiantes que integran el club, en su mayoría, cuentan con la compañía de papá y mamá; sin embargo, por las condiciones socioeconómicas (estratos 1 y 2) los dos padres están obligados a trabajar, ya sea en actividades formales o informales, por lo tanto, el tiempo que pasan con ellos en jornada extraescolar es poco. Este tiempo, en el mejor de los casos, es acompañado por abuelos o tías, pero es más común que los estudiantes estén solos.

Los estudiantes que se vinculan a esta experiencia tienen como característica común su curiosidad e interés por la astronomía, pero también son claras sus particularidades cognitivas y de convivencia. Algunos de ellos tienen niveles académicos sobresalientes, otros presentan dificultades en los procesos de descripción, interpretación, argumentación y proposición o problemas para cumplir con las actividades escolares (tareas, trabajos, entre otros). Igualmente, se vinculan tanto niños disciplinados como aquellos que tienen inconvenientes en las relaciones con los demás y poca asertividad para solucionar los conflictos.

\section{El porqué de una experiencia de innovación}

La fascinación por el cielo nocturno, por las estrellas, por Saturno y sus anillos, entre otros fenómenos, genera en los niños preguntas sobre el universo, y aprovechan cualquier oportunidad para aprender sobre esto; sin embargo, son temas relegados a las ciencias naturales o sociales y son abordados, en el mejor de los casos, de acuerdo al interés o conocimiento del maestro encargado de orientar estas áreas. Igualmente, la mayoría de las prácticas escolares abordan el conocimiento por especialidades y, en consecuencia, el estudiante presenta dificultades al vincular los elementos que cada área le ofrece. La astronomía, "la madre de todas las ciencias, al ser una ciencia interdisciplinaria por excelencia" (Meléndez, 2002, párr. 1), al concertar la especificidad de tantas áreas del conocimiento y al mostrar un panorama distinto de las ciencias, se constituye en una alternativa para que los estudiantes relacionen esos saberes obtenidos en el proceso de formación, acercándolos al conocimiento de una forma holística. 
Es una experiencia que trabaja bajo la modalidad de club, porque se considera una estrategia organizativa que, sin alejarse del contexto académico, integra bajo un interés común tanto a estudiantes de diferentes grados de escolaridad como a maestros de diferentes disciplinas y facilita el desarrollo de un ambiente de aprendizaje colaborativo entre sus integrantes.

Por la metodología implementada, esta experiencia permite que cada integrante reconozca los conocimientos que posee, indague, formule preguntas sobre un fenómeno, busque y discuta cómo obtener información para contrastar esas ideas originales con sus nuevos aprendizajes (Devés \& Reyes, 2007) y que, como resultado de su proceso de formación en el club, desarrolle proyectos de investigación de índole escolar.

Ha sido una experiencia que con el tiempo ha sufrido cambios; por lo tanto, se espera que los maestros continúen aportándole desde el diseño de estrategias didácticas y, conforme a los procesos de retroalimentación, se consolide aún más la propuesta.

\section{Los propósitos}

El propósito general se ha definido como: utilizar la interdisciplinariedad de la astronomía mediante la proposición y realización de proyectos de investigación de tipo escolar, que evidencien el desarrollo de habilidades de pensamiento y la vinculación de los aprendizajes alcanzados en el proceso por parte de los estudiantes integrantes del club.

Para alcanzarlo, se han propuesto otros adicionales:

- Implementar estrategias didácticas que permitan la comprensión y apropiación de los conceptos propios de la astronomía.

- Acompañar en el diseño y ejecución de proyectos de investigación de tipo escolar a los estudiantes con mayor trayectoria en el club (nivel experto).
- Participar en actividades académicas locales, nacionales e internacionales, que fortalezcan la formación de los integrantes y su reconocimiento con los proyectos realizados por ellos.

- Gestionar el acompañamiento de instituciones especializadas en el proceso de formación de los estudiantes.

- Vincular a los niños de ciclo I, mediante actividades lúdicas que favorezcan su acercamiento a la astronomía.

\section{Su implementación}

Desde el año 2009, para facilitar la planeación, el desarrollo, la revisión y la evaluación de los procesos, la experiencia se estructura en cinco etapas anuales: Motivación, Identificación de intereses, Fundamentación, Proyección y Evaluación; a su vez, la etapa de Fundamentación está organizada en cinco fases que ponen de manifiesto el enfoque metodológico asumido en esta propuesta. Cada etapa tiene una intencionalidad e involucra unas acciones orientadas al alcance de las metas anuales (figura 1) y el logro de los propósitos, así: Motivación, punto de partida cuya característica es el ser continua; Identificación de intereses, donde se definen las metas del año; Fundamentación, corresponde a las acciones; Proyección, atañe a la verificación de los procesos; y Evaluación, que genera los ajustes para realizar la nueva planeación. 


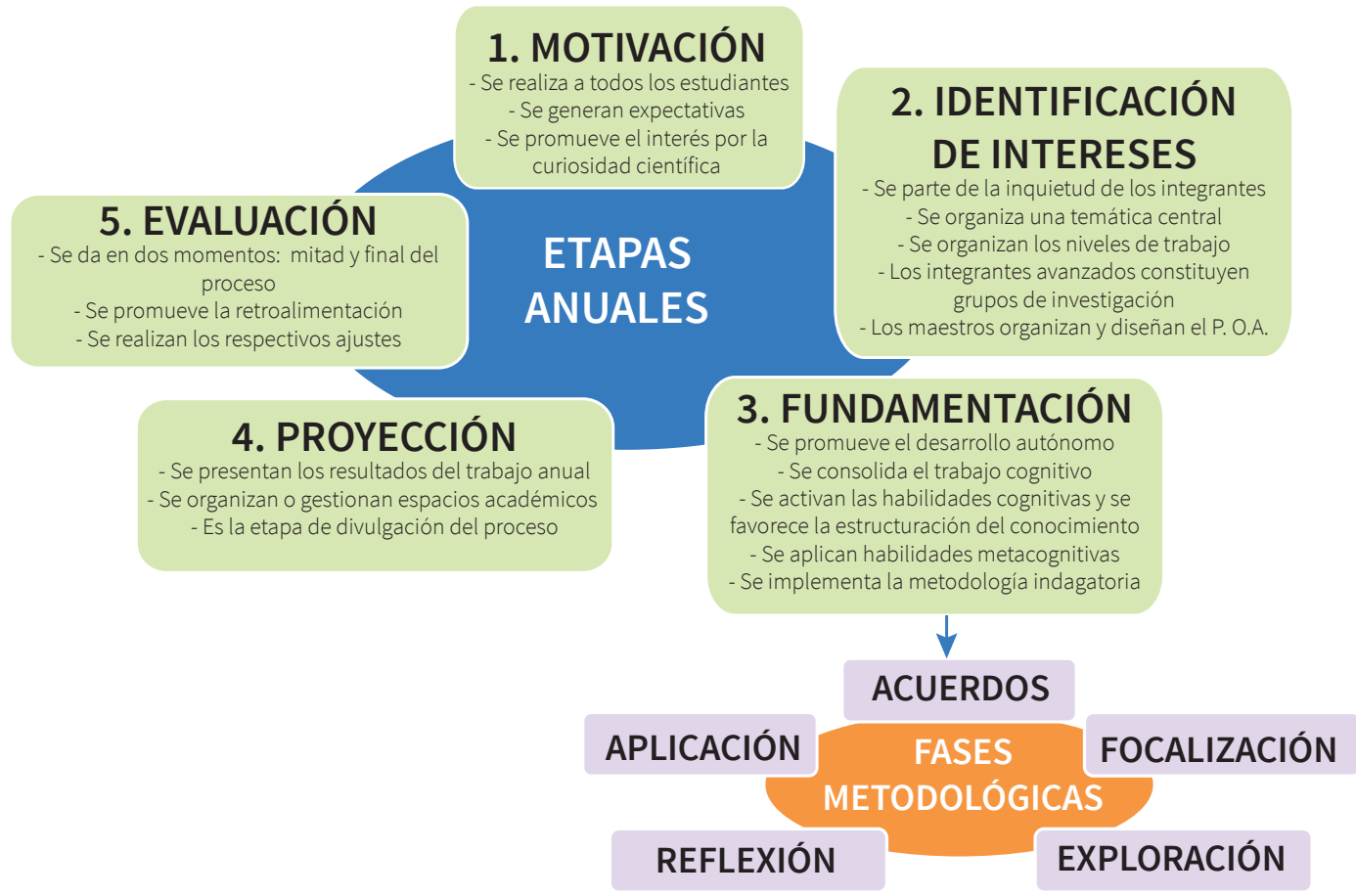

Figura 1. Acciones que involucran las etapas anuales

Inicialmente, la etapa de Fundamentación solo se orientaba desde el desarrollo de talleres, luego se incorporó el trabajo cooperativo y posteriormente, se incluyó el trabajo por proyectos. Al ser la etapa en la que convergen la construcción, la reconstrucción o consolidación del conocimiento y el momento en el que se trasciende la forma como se accede a él y como se enseña, desde el 2011 se decidió abordar los principios básicos de la metodología basada en la indagación, apoyada con el trabajo por proyectos, el aprendizaje autónomo y el trabajo cooperativo. No obstante, por las características de los temas que se tratan en el club, las fases que plantea la metodología se asumen con ajustes que los maestros van realizando. En la figura 2 se observan los ajustes realizados:

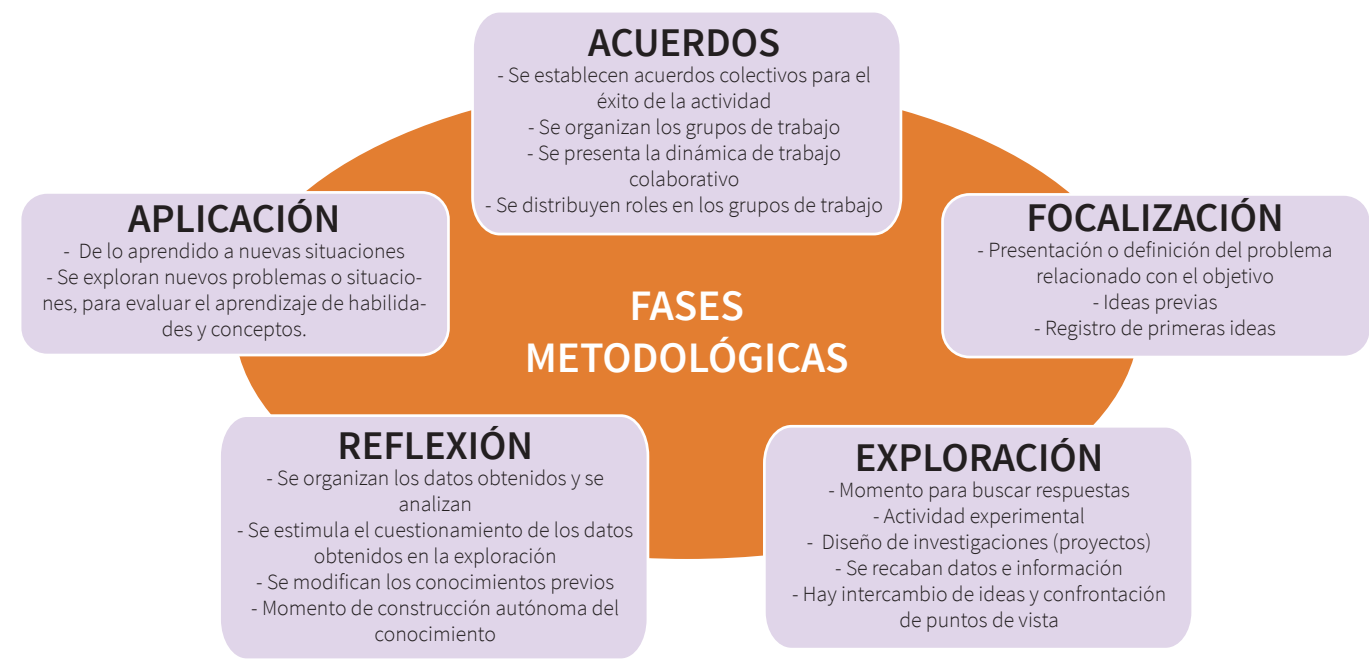

Figura 2. Aspectos que aborda cada fase de la metodología implementada en la etapa de Fundamentación 
La enseñanza de la astronomía debe ir más allá de mostrar o describir los fenómenos suscitados en el universo. Por lo anterior, la metodología propuesta ha requerido del diseño e implementación de diferentes estrategias didácticas, conducentes a generar un avance significativo en los aprendizajes sobre temas de astronomía, sin olvidar la rigurosidad que esta requiere y la vinculación de los saberes que en ella se conjugan.
Entre tales estrategias están: la implementación de talleres, foros, conferencias y salidas de observación; la elaboración de escritos y de bitácoras, y la preparación de exposiciones para eventos académicos.

La tabla 1 presenta las estrategias utilizadas, categorizándolas de acuerdo con la intención con la cual se implementan:

Tabla 1. Estrategias didácticas implementadas en la enseñanza de la astronomía

\begin{tabular}{|c|c|c|c|}
\hline \multicolumn{4}{|c|}{ ESTRATEGIAS } \\
\hline \multicolumn{2}{|c|}{$\begin{array}{l}\text { ESTRATEGIAS DIDÁCTICAS DE TIPO PEDAGÓGICO: } \\
\text { Acciones que permiten acceder, conceptualizar y comprender los } \\
\text { temas propios de las disciplina }\end{array}$} & \multirow{2}{*}{\multicolumn{2}{|c|}{$\begin{array}{l}\text { ESTRATEGIAS DIDÁCTICAS DE TIPO } \\
\text { COMUNICATIVO: } \\
\text { Acciones que promueven el desarrollo de } \\
\text { competencias propias de la escritura }\end{array}$}} \\
\hline Fundamentación teórica & Trabajo de campo & & \\
\hline Talleres & Observaciones astronómicas & Diario del astrónomo & Medios escolares \\
\hline $\begin{array}{l}\text { Su finalidad es motivar, incentivar } \\
\text { y planear el trabajo. Favorece la } \\
\text { exposición de ideas, el consenso y } \\
\text { la planificación en grupo. } \\
\text { Involucran estrategias adicionales } \\
\text { como la lúdica, el modelado, } \\
\text { las analogías, los vídeos, las } \\
\text { conferencias. }\end{array}$ & $\begin{array}{c}\text { El trabajo de observación } \\
\text { en astronomía, } \\
\text { se completa con } \\
\text { la observación } \\
\text { práctica directa, por } \\
\text { ello se programan } \\
\text { observaciones } \\
\text { nocturnas o el uso de } \\
\text { software astronómico. }\end{array}$ & $\begin{array}{l}\text { La bitácora } \\
\text { además de ser } \\
\text { un instrumento } \\
\text { de registro, es } \\
\text { una herramienta } \\
\text { que favorece la } \\
\text { comunicación } \\
\text { interna y } \\
\text { externa. }\end{array}$ & $\begin{array}{l}\text { Dar a conocer } \\
\text { lo que sabemos } \\
\text { y mantener un } \\
\text { vínculo con } \\
\text { los otros es } \\
\text { importante, con } \\
\text { ese propósito } \\
\text { se diseñan } \\
\text { y utilizan } \\
\text { medios de }\end{array}$ \\
\hline \multicolumn{2}{|c|}{ Diseño y ejecución de proyectos } & & \\
\hline \multicolumn{2}{|c|}{$\begin{array}{c}\text { Es el resultado del proceso de aprendizaje. Son diseñados y } \\
\text { desarrollados por los estudiantes del nivel Expertos, a partir de } \\
\text { una pregunta o tema de interés. Se promueve el proceso de } \\
\text { investigación. }\end{array}$} & & $\begin{array}{c}\text { la emisora y el } \\
\text { periódico. }\end{array}$ \\
\hline \multicolumn{2}{|c|}{ Diario del astrónomo } & & \\
\hline \multicolumn{2}{|c|}{$\begin{array}{c}\text { La bitácora es el registro sistemático de cada una de las experiencias } \\
\text { vividas por los integrantes. }\end{array}$} & & \\
\hline \multicolumn{4}{|c|}{ Módulo astronómico } \\
\hline
\end{tabular}

Cada año se vinculan nuevos estudiantes y se integran con los compañeros que ya están en el proceso, con el fin de favorecer el intercambio de saberes; pero, sucedió que al momento de retomar temáticas imprescindibles para los noveles hubo desmotivación en los integrantes antiguos. Así, en el 2012, se consolidó una organización por niveles y se diseñó un plan de aprendizaje con actividades que respondieran a la estructura mental de los integrantes, a sus procesos de pensamiento y a su trayectoria en el club. 


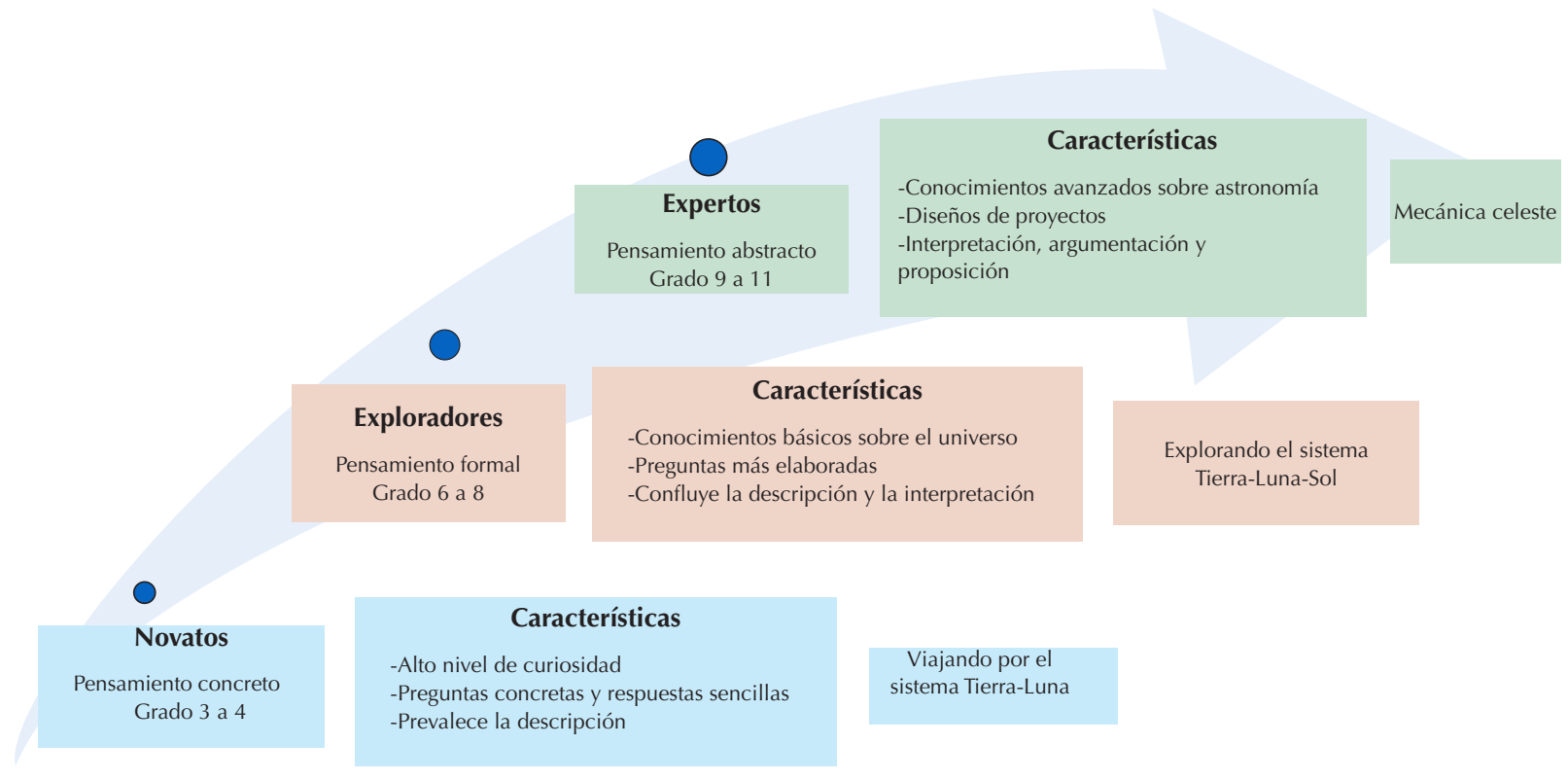

Figura 3. Estructura organizativa del club y ejemplo del plan de aprendizaje que se aborda en cada nivel

La intención no es curricularizar la astronomía, pues uno de los aciertos de este trabajo es que se aborda desde los intereses de los estudiantes. Aun así, esta estrategia ha permitido organizar de forma sistémica los temas y mantener un vínculo entre lo precedido y lo posterior en el proceso de formación, brindando coherencia a los contenidos que serán utilizados por los estudiantes en sus proyectos de trabajo; sin embargo, en ocasiones se organizan actividades que involucran a todos los integrantes independientemente del nivel.

\section{Los hallazgos}

\section{Transformaciones pedagógicas en la práctica de los maestros}

\section{Diseño de plantilla de planeación de talleres}

La reflexión pedagógica ha llevado a realizar ajustes a la metodología abordada, como respuesta a las particularidades y las necesidades de la experiencia. Por ejemplo, la metodología ECBI (Enseñanza de las Ciencias basada en la Indagación) propone en la fase de exploración realizar actividades experimentales con el fin de verificar las ideas previas del estudiante (Harlen, 2011), pero como se trabajan temas donde lo tangible no siempre está presente y la experimentación no es fácil, fue necesario innovar utilizando estrategias didácticas como lecturas, canciones, videos, analogías y adaptarlas para confrontar el saber inicial de los estudiantes con el saber construido por la comunidad científica. Asimismo, se incorporó una fase inicial Ilamada Acuerdos, como ejercicio introductorio al trabajo de formación. Esto se ha materializado en una plantilla diseñada para orientar y mantener los lineamientos propuestos por la metodología al momento de planear los talleres.

Al diseñar los talleres se establece la relación de conocimientos entre las diferentes áreas, ya que por las prácticas escolares en las que el saber es parcializado por especialidades, en los estudiantes surge el conflicto de relacionar los elementos que cada disciplina le brinda a la astronomía. Por esto, como lo manifiesta Meléndez (2002), al ser la astronomía una ciencia interdisciplinaria, uno de los propósitos en el ejercicio de formación de los integrantes es concertar la especificidad de tantas áreas y su utilidad en la comprensión de los fenómenos del universo. Como resultado del trabajo realizado en los talleres, los estudiantes han evidenciado la cooperación entre las diversas disciplinas del conocimiento humano, siendo abiertos a las contribuciones que estas traen a su proceso de formación, lo cual se refleja en los proyectos que realizan, en especial los que se encuentran en el nivel de expertos. 


\section{El taller, estrategia didáctica por excelencia}

Como particularidad, la astronomía invita a conocer lo que ella encierra, se constituye en una herramienta importante para la construcción del conocimiento. Pero, como lo expresa González (2008) el proceso de enseñanza requiere que el maestro organice el ambiente de aprendizaje, sea guía en este ejercicio pedagógico y estimule al estudiante, es decir, requiere de una forma de enseñar para que él aprenda mejor y más fácil, acudiendo a diferentes estrategias didácticas, sin perder la rigurosidad de la ciencia.

El taller ha sido la estrategia didáctica esencial en el proceso de fundamentación realizado con los estudiantes, con este se ha logrado que trasciendan en su forma de exponer ideas. Ya no se quedan en la memorización del concepto o fenómeno y de cómo ocurre, sino que ahora explican por qué ocurre y para qué lo aprenden; en otras palabras, han trascendido del conocimiento demostrativo y procedimental, al conocimiento esquemático y estratégico ${ }^{5}$. Es de precisar que esto se evidencia con los proyectos realizados por los integrantes que llevan un proceso de varios años en el club -nivel de expertos- y la respectiva exposición en diferentes eventos de tipo académico.

Son muchos los talleres abordados que han arrojado resultados positivos, pero igualmente se les han realizado mejoras con el fin de fortalecer el proceso de formación con los estudiantes, ya que en un principio involucraban un alto porcentaje de conferencias magistrales que no permitían la construcción del conocimiento y quedaban vacíos conceptuales.

\section{Transformaciones en los estudiantes}

\section{Del trabajo individual al trabajo cooperativo}

Se ha evidenciado que vincular a estudiantes de diferentes niveles escolares permite un diálogo entre los de mayor trayectoria con los recién ingresados y favorece los procesos de convivencia al promover la solidaridad, el respeto, el reconocimiento e identificación con el par. Sin embargo, al momento de desarrollar los talleres

5 Premisa consolidada a partir de la participación en el programa Pequeños Científicos ofrecido por la Universidad de los Andes. se convierte en un inconveniente porque los que llevan más tiempo desean aprender otros temas y los pequeños requieren ese conocimiento que ya se ha abordado. Por lo anterior, en distintas oportunidades se les da la responsabilidad a los estudiantes del nivel de expertos de diseñar y desarrollar talleres para sus compañeros novatos, lo cual favorece las actitudes de liderazgo y la identificación del estado de su proceso cognoscitivo y actitudinal como resultado de su vinculación al club.

De la implementación de los talleres y como aplicación del trabajo cooperativo, los estudiantes reconocen un objetivo común que motiva al grupo a trabajar conjuntamente para alcanzarlo, existen responsabilidades para el éxito del mismo y hay comunicación asertiva, proporcionándoles habilidades que les ayudan a interactuar con sus pares, a la vez que les brindan destrezas para construir, descubrir, transformar y ampliar los contenidos conceptuales (González \& García, 2007); no obstante, es necesario continuar fortaleciendo esta modalidad de trabajo, porque aún encontramos estudiantes a quienes se les dificulta relacionarse con los integrantes noveles de menor edad y cumplir con los compromisos asignados.

\section{De la práctica al trabajo de campo}

El trabajo de fundamentación se resalta al complementarlo con el trabajo de campo; es el ejercicio práctico que motiva y reafirma en los integrantes su gusto por la astronomía, es la observación directa de algunos de los fenómenos que se han abordado en los talleres. Se ha tenido la oportunidad de realizar salidas de observación a Villa de Leyva, al desierto de La Tatacoa y Sesquilé, las cuales han arrojado resultados positivos, por ello se constituyen como los momentos en que se justifica lo trabajado, no solo desde la astronomía, sino desde todas las áreas del conocimiento, incluyendo aspectos sociales, ambientales y de convivencia.

\section{La escritura y registro de lo aprendido o de las experiencias vividas}

Es sabido que, en el contexto escolar, la escritura es una de las acciones poco favoritas de los estudiantes; sin embargo, en el conocimiento de las ciencias la lectura y la escritura son fundamentales. Desde el club se promueve, a partir del 2009, el registro de las acciones realizadas en los talleres en una herramienta de sistematización Ilamada Diario del Astrónomo; 
cada integrante en su diario escribe, utilizando el estilo deseado, las experiencias vividas y el aprendizaje alcanzado. Aunque la apropiación de esta herramienta no ha sido fácil debido al poco interés por la escritura, se han evidenciado transformaciones en los integrantes como resultado de los procesos de formación y el tiempo de vinculación al club.

Por otro lado, la institución cuenta con medios de información como la emisora y el periódico escolar, por eso desde el club se promueve su utilización con escritos realizados por los estudiantes con información relevante sobre temas de astronomía o que den cuenta de las experiencias vividas por ellos. Se ha logrado que algunos escriban artículos para ser publicados y, dependiendo del nivel y el tiempo de participación en el club, se ve el grado de complejidad en sus escritos, entre los que se encuentran artículos de tipo descriptivo y otros propositivos.

\section{Consolidación del proceso de formación}

La estrategia por proyectos se caracteriza porque tiene en cuenta los intereses de los estudiantes, responde a problemas, preguntas e hipótesis, reconoce la utilidad del conocimiento, posibilita el trabajo cooperativo, confronta conceptualizaciones, corrige interpretaciones y complementa informaciones (Bueno, 2002, p. 65); en este sentido, los integrantes del nivel expertos conforman colectivos de trabajo para diseñar y realizar ejercicios de investigación de tipo escolar, a partir de un interrogante que les que surge durante el proceso de fundamentación. Como lo expresa Cerda (2001), con el desarrollo de estos ejercicios los estudiantes han mostrado un cambio conceptual en diferentes tópicos de la astronomía y se han formado en la autonomía, lo cual se evidencia con los proyectos realizados.

Al incluir el trabajo por proyectos, se favorece el aprendizaje autónomo porque se estimula a los estudiantes a desarrollar actitudes, aptitudes y habilidades para tomar decisiones acertadas (Chica, 2010, p. 193) y dar soluciones a problemas a partir del impulso de proyectos de interés. Se logra así, que sean autores de su propio conocimiento. En esta dinámica, el maestro cumple el papel de mediador con el diseño de actividades y con el acompañamiento de los procesos de investigación escolar realizados por los integrantes con mayor trayectoria.
Dependiendo del estado de los proyectos realizados por los estudiantes, han sido inscritos en diferentes eventos académicos en el ámbito local, distrital y nacional, con el fin de socializar el conocimiento alcanzado en el ejercicio de fundamentación y para consolidar su proceso de formación en el club. En estas socializaciones, además de evidenciar los niveles de apropiación del conocimiento, se han fortalecido las competencias comunicativas, lo cual se manifiesta en la claridad y coherencia expositiva; así mismo, la exposición de sus proyectos en eventos de esta índole ha contribuido a que ellos reconozcan la importancia de su trabajo y la validación por parte de la comunidad a partir de la divulgación, elementos importantes en el proceso científico. Como ejemplo, se participó en un concurso de Astronomía donde los estudiantes pudieron evidenciar sus dificultades y sus competencias, allí, uno de los estudiantes de grado noveno, a pesar de competir con estudiantes de grado undécimo de otras instituciones, alcanzó un buen nivel.

Se ha participado, con los proyectos de los estudiantes, en ferias de las ciencias y foros locales, en eventos nacionales como Expociencia y Expotecnología Juvenil, en las Ferias de Astronomía y ciencias afines organizadas por el Planetario Distrital y en el I Encuentro de Talentos del Distrito, organizado por la Red de Talentos de la SED; también en eventos internacionales como Catch Star y Ciencia en Acción.

\section{Del colegio a otros espacios de formación}

Trascender el aula y el colegio en el proceso de formación ha sido un propósito permanente en la acción de los maestros hacedores de esta propuesta; por ello, se ha vinculado el club a instituciones que apoyen y fortalezcan este proceso.

En el año 2004, el club se vinculó de manera formal al trabajo realizado por el Planetario Distrital; de esta manera, con el apoyo de talleristas de esta institución y con la participación en las diferentes actividades organizadas por ellos (ferias, proyecciones, campamentos espaciales, encuentros entre clubes, encuentro de líderes estudiantes de clubes, talleres, conferencias, encuentro con expertos en diferentes áreas de la astronomía), se ha logrado fortalecer el proceso de fundamentación de los integrantes del club (maestros y estudiantes). Los estudiantes han cambiado su percepción frente a sus capacidades y responsabilidades, 
al representar al club dando a conocer su experiencia en el mismo y los aprendizajes consolidados en los diferentes proyectos realizados por ellos. Esto se revierte en los integrantes noveles pues evidencian las potencialidades que pueden desarrollar y los espacios que pueden conquistar.

En los años 2008 y 2009, Maloka, desde el programa Ondas, realizó una convocatoria para vincular proyectos en la línea ambiental y robótica respectivamente, lo cual se consideró una oportunidad para fortalecer el proceso de formación de los integrantes en el ámbito de la investigación.

En 2011, el club se vincula a la iniciativa internacional Misión X: Entrénate como un astronauta, implementada por la NASA (National Aeronautics and Space Administration), el IGAC (Instituto Geográfico Agustín Codazzi) y la CEE (Comisión Colombiana del Espacio) para que los estudiantes, en una serie de desafíos, experimentaran los requisitos en cuanto a aptitudes físicas y necesidades nutricionales que requiere un astronauta al entrenarse para desarrollar su labor.

\section{Vinculación de los niños de ciclo I}

En 2013, se decide vincular a los niños del ciclo I e implementar con ellos el juego como un ingrediente en los talleres, la intención de este proceso fue explorar el talento y cultivar la curiosidad innata que tienen los niños por la astronomía. Se trabaja desde el juego porque, como lo expresa Benítez (2009), este tiene características que, desde el proceso educativo, fortalece en los niños la comunicación y su dimensión física y mental; desarrolla su inteligencia emocional, así como su personalidad, y enriquece tanto la imaginación como la creatividad. En consecuencia, desde la planeación misma de los talleres, el juego ha sido indispensable en cada uno de ellos ${ }^{6}$. Al trabajar con los niños de este ciclo, se evidencia su motivación, su inquietud y curiosidad, se identifican niños que tienen gran interés por la astronomía y que a pesar de su edad, conocen sobre el tema.

6 El juego no es una acción nueva para el trabajo del club, ya que en el diseño de los talleres se ha propendido porque en las actividades desarrolladas esté presente la lúdica, pues sabemos por experiencia que, si el proceso de aprendizaje se hace divertido, el acceso al conocimiento se facilitará.

\section{Reflexión final}

La metodología implementada y las estrategias didácticas definidas han favorecido el acercamiento de forma dinámica y autónoma al conocimiento y apropiación de la disciplina, lo cual se evidencia en los proyectos desarrollados y presentados por los estudiantes en diferentes espacios académicos y concursos.

Se han generado aprendizajes nuevos en los integrantes los cuales han incidido positivamente en competencias académicas y en actitudes como la expresión, la responsabilidad y la solidaridad, que se evidencian en el liderazgo que asumen los estudiantes cuando se encuentran en el nivel de Expertos.

Como resultado de la constancia y el proceso generado durante los años de desarrollo de la propuesta, se cuenta con el acompañamiento permanente de instituciones como el Planetario Distrital; asimismo, al participar con los proyectos de los estudiantes, se ha logrado la asesoría de Maloka y la vinculación al Programa Ondas, línea ambiental y línea de robótica.

El trabajo por proyectos ha favorecido el acercamiento de forma dinámica a las ciencias naturales, la matemática, la física, las ciencias sociales, el arte, entre otras, lo cual se evidencia en los proyectos propuestos y desarrollados por los estudiantes.

El interés por el conocimiento del universo se ha aprovechado en tiempos distintos al escolar, siendo una estrategia que invita al aprovechamiento del tiempo libre.

En síntesis, como lo expresa Puerta (2008), la edad escolar es definitiva en el desarrollo de valores, es la etapa donde se alcanza a moldear lo que el hombre será en el futuro; por ende, si como maestros aprovechamos la curiosidad de los pequeños por los fenómenos del universo y consolidamos propuestas que muestren la astronomía con toda su magia, desarroIlando actividades didácticas tendientes a estimular el descubrimiento de la relación entre los objetos celestes y su lugar en el universo, contribuiremos a perfilar el camino profesional que tomarán nuestros estudiantes. El aprovechar sus preguntas, sus observaciones, sus predicciones, su interés por la astronomía, además de promover la ciencia, la investigación y la cultura, favorecerá su formación, le permitirá entender el mundo en que vive, y le ayudará a comprender su herencia cultural y su diversidad. 


\section{Referencias}

Benítez, M. (2009). El juego como herramienta de aprendizaje. Revista Digital Innovación y Experiencias Educativas, 16. ISSN 1988-6047. Recuperado el 12 de agosto de 2013, de http:// www.csi-csif.es/andalucia/modules/mod_ense/revista/pdf/ Numero_16/MARIA\%20ISABEL_BENITEZ_1.pdf

Bueno, L. (2002). La enriquecedora experiencia de asesorar la planeación y puesta en marcha de proyectos de aula. En L. Bueno (ed.). Proyectos de Aula con base en la experimentación y la interdisciplinariedad. (pp. 65-70). Bogotá, Colombia: Universidad Nacional.

Cerda, H. (2001). Proyectos de Aula. Bogotá, Colombia: Cooperativa Editorial Magisterio.

Chica, F. (2010). Factores de la enseñanza que favorecen el aprendizaje autónomo. Portada de Revistas. Recuperado 11 de agosto de 2013, de dialnet.unirioja.es/descarga/articulo/3709190.pdf

Devés, R. \& Reyes, P. (2007). Principios y estrategias del programa de educación en ciencias basada en la indagación (ECBI).
Pensamiento Educativo, 41, (2). Recuperado 12 de agosto de 2013, de http://pensamientoeducativo.uc.cl/index.php/pel

González, D. (2008). Didáctica o dirección del aprendizaje. Bogotá, Colombia: Cooperativa Editorial Magisterio.

González, N. \& García, R. (2007) El Aprendizaje Cooperativo como estrategia de Enseñanza-Aprendizaje en Psicopedagogía (UC): repercusiones y valoraciones de los estudiantes. Revista Iberoamericana de Educación. Recuperado el 26 de abril de 2013, de http://www.rieoei.org/expe/1723Fernandez.pdf.

Harlen, W. (2011). Aprendizaje y enseñanza de ciencias basadas en la indagación. En J. Campos, C. Montecinos \& A. González (Eds.). Mejoramiento escolar en acción (pp. 33-48). Chile: Salesianos Impresores.

Meléndez, J. (2002). Astronomía: Ciencia Interdisciplinar. Boletín CSI Consejo Superior de Investigaciones, 45. Recuperado el 2 de febrero de 2012, de http://sisbib.unmsm.edu.pe/bibvirtualdata/ publicaciones/consejo/boletin45

Puerta, G. (2008). Astronomía para todos. Bogotá, Colombia: Panamericana.

\section{Diálogo del conocimiento}

En el país se han conformado, desde hace varios años, clubes de astronomía que desarrollan proyectos con estudiantes de bachillerato. Es el caso de un colectivo de entusiastas, en Medellín, entre astrónomos, aficionados y docentes universitarios, quienes han compartido experiencias pedagógicas en el marco del proyecto "Maestros gestores de nuevos caminos" orientado y promovido por la Corporación Región, con el apoyo de Confiar.

Es indudable el impacto positivo que dichas iniciativas tienen en niños y jóvenes, quienes, interesados en conocer muestran gran disposición y esfuerzo por "aprender" -y, de cierta manera,- entrar en contacto con lo que "desconocen". Sin embargo, los docentes persistimos en las violencias para imponer el aprendizaje, que en últimas constituyen modelos canónicos que obligan a los pequeños a prestar atención a lo que, como profesores, consideramos "bueno" para ellos, lo cual termina por menoscabar su voluntad y subordinarla a la nuestra.

Es ensombrecedor confirmar que no hemos querido hacer caso de los desarrollos más actuales de la pedagogía y de la psicología infantil, desde donde se nos reitera que los niños tienen intereses diversos y aprenden de formas diferentes.

Como maestros estamos obligados a buscar, incesantemente, una educación que atienda a los verdaderos intereses y las necesidades de nuestros educandos. En ese sentido, propuestas como las de Kathy Short ("El aprendizaje a través de la indagación"), y Laurence Splitter y Ann Sharp ("La otra educación"), son pertinentes en la actualidad.

Sin más contexto, este artículo es pertinente, sobre todo, para el debate educativo actual, cuando las directrices de la OCDE, cada vez más frenteras, apuntan a reducir la enseñanza a los campos que se consideran funcionales dentro del modelo económico. Aquí cabe recordar la reorganización curricular por ciclos y los campos de pensamiento: la primera busca atender el avance del conocimiento sobre los procesos cognitivos que se dan durante el tiempo escolar, para así obtener una mejor correspondencia en ello y restarle notoriedad a la arbitrariedad de la escuela. En los segundos, la interdisciplinariedad hace comprensible la realidad y facilita una intervención más responsable en la construcción de la vida humana. De esa búsqueda, en el texto se percibe la pertinencia como el hilo conductor de una vida humana con un significado más allá del juego de la acumulación como supuesta garantía -manifiesto engaño- de la sobrevivencia de la especie. Esta visión holística, que asume todos los aspectos que nos constituyen como especie y determinan la magnitud de lo que somos respecto a la totalidad de lo que es, puede ayudar a que, en el tiempo, la humanidad se oriente hacia caminos opuestos a los que hoy transita.

Fernando Escobar 